\title{
Verification and Implementation of Pseudo-Random-Binary-Sequences for Online Determination of Grid Impedance Spectrum
}

\author{
Udit Tewari ${ }^{1}$, Surena Neshvad ${ }^{2}$, Daniel Goldbach ${ }^{1}$ and Jürgen Sachau ${ }^{2}$ \\ ${ }^{1}$ Faculty of Energy Technology \\ FH Aachen, Campus Jülich \\ Jülich, Germany \\ e-mail: Udit.Tewari@alumni.fh-aachen.de, Goldbach@fh-aachen.de \\ ${ }^{2}$ Interdisciplinary Centre for Security Reliability and Trust (SnT) \\ University of Luxembourg \\ Luxembourg City, Luxembourg \\ e-mail: Surena.Neshvad@uni.lu, Juergen.Sachau@uni.lu
}

\begin{abstract}
Renewable energy technologies as Distributed Energy Sources (DER) connected via power inverter on medium or low voltage grid are expected to become a more important part of future generation system. Therefore, it has to be ensured that their integration doesn't jeopardize the stability and performance of the power system which can only be attained with improved monitoring and control of system parameters on real time basis such as the equivalent grid impedance seen from the grid-tied DER. In order to ensure this, this paper investigates a reliable and precise method for online equivalent grid impedance estimation based on Pseudo-Random-BinarySequences (PRBS), which has been extensively used in system identification, communication and information theory. It allows real time computation of complete spectrum of grid impedance at the Point of Common Coupling (PCC) by injecting broad spectrum identification patterns of harmonics and interharmonics. The impedance spectrum thus, can be used for filter design, power quality evaluations, grid status determination and inverter tuning. In this paper, a PRBS-based active identification method is tested and implemented on an off-grid system consisting of a 3 phase inverter prototype developed in the lab and has been used to identify the grid impedance spectrum of a 3 phase purely inductive load connected at the output of the inverter. The results obtained confirm that the method estimates the grid impedance spectrum over a significant frequency range with high resolution on real time basis.
\end{abstract}

\section{Key words}

Inverter, Pulse Width Modulation (PWM), PseudoRandom-Binary-Sequences, Grid Impedance Spectrum

\section{Introduction}

The world's collective capacity to generate power through renewable energy sources has seen a significant growth in the last few decades. These sources are considered to have a long term potential to meet the entire world's energy demand and an effective way to reconfigure the present energy supply structure while at the same time achieving reduction in global $\mathrm{CO}_{2}$ emissions. Also constant improvement in cost and efficiency and their use as DER has reduced costs per kWh. The Medium-Term Renewable Energy Market Report by the International Energy Agency (IEA) published in August 2014 shows that energy from renewable energy sources like Wind, Solar and Hydro will likely rise to 26 percent by 2020 which is currently 22 percent of the world's electricity [1]. In this context large part of renewable energy sources, mainly wind and photovoltaic (PV) energy, has been used as inverter based distributed power generators integrated to the medium voltage grid or low voltage grid.Increased use of such systems in future will therefore increase the complexity of the grid and will require advanced and intelligent monitoring and observation tools in order to integrate them harmoniously and effectively to the grid. In order to address these concerns, the equivalent grid impedance seen from the inverter can be considered an important parameter that will allow the device to react instantaneously to critical conditions and to correct its behaviour. It can be used to determine properties of the grid that are hard to detect such as islanding conditions [2], resonance frequencies in the grid and major harmonics induced by load capacitance [3] and voltage stability of the grid-tie inverter [4]. It can also be used for inverter droop control optimization, by adjusting the inverter output voltage amplitude and frequency for controlling active and reactive power [5].

This paper reviews the grid impedance spectrum identification technique proposed and presented in [8], and describes its implementation on an off-grid system consisting of a 3 phase PWM inverter prototype developed at SnT Netpower Laboratory in order to verify its performance in real settings and to determine the grid impedance spectrum of the 3 phase load connected at the output of the inverter. The paper is structured as follows: Section 2 presents a brief review of PRBS and its properties presented in [8]. Section 3 describes the PRBS implementation on PWM and the method of determination 
of grid impedance spectrum identification by PRBS injection. The complete test setup implemented and used in order to verify the performance in real settings is presented in Section 4. Section 5 illustrates and discusses the results obtained.

\section{PRBS and their properties}

Pseudo-Random-Binary-Sequences also known as pseudo-noise (PN), linear feedback shift register (LFSR) sequences or maximal length binary sequences (msequences) [6] are random bit streams of ' 1 's or ' 0 's that repeatthemselves. This is a desirable feature for identification as it allows predicting of an incoming sequence and it also makes it possible to register and count errors that might occur in the sequence. A PRBS bit stream can be generated by using a linear feedback shift register (LFSR) which is a series of shift registers, combined with logical XOR gates. Three parameters characterize the sequence of bits it produces: the number of bits L, the tap position, and the initial seed (sequence of bits that initializes the register). An L-bit LFSR generates $2^{\mathrm{L}}-1$ bits of PRBS. The power spectrum of the PRBS sequence has $\operatorname{an} \frac{\sin ^{2} x}{x^{2}}$ envelope [6].

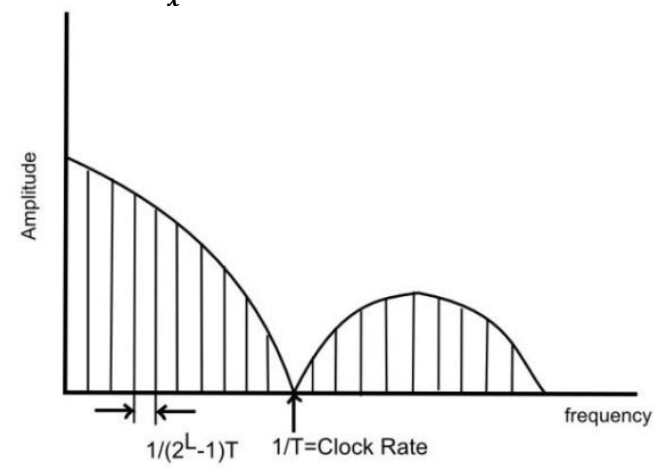

Figure 1: Power spectrum for PRBS sequence

The nulls in the power spectrum occur at $\boldsymbol{f}=\frac{\boldsymbol{n}}{\boldsymbol{T}}$, where T is the bit duration and $\mathrm{n}$ is the integer. From 0 to $\frac{\mathbf{1}}{\boldsymbol{T}}$ the spectrum contains all the line frequencies with the spacing of $\frac{1}{\left(2^{L}-1\right) T} \mathrm{~Hz}$ where L is the L-bit LFSR [6]. Thus, the spectrum of PRBS is discrete but by careful optimization between maximal length, sampling frequency and PRBS bit frequency, a white noise like spectrum for a certain frequency range can be obtained. A high amplitude spectrum is necessary in order to achieve optimal energy distribution and accuracy for impedance spectrum measurement. In addition, the logic can also be easily implemented on a digital controller with shift registers and XOR gates and can be easily reconfigured depending upon the spectrum desired. Thus, a short sequence can be produced for coarse estimation, and a longer one for more refined spectrum [8].

\section{PRBS Implementation on PWM and Grid Impedance identification}

Pulse width modulation (PWM) technique is the heart of most inverter signal generation systems and aims to generate a signal, which after some filtering would result in a good quality sinusoidal waveform of desired fundamental frequency and magnitude. Generation of the desired signal is achieved by comparing the desired reference waveform (modulating signal) with the high frequency triangular 'carrier' wave. The IGBTs are turned ON or OFF depending on whether the output is set to High or Low.

In order to implement the PRBS, the carrier wave is modified in a manner so that the PRBS naturally overlaps with the reference signal used for PWM generation. Each carrier cycle is thus assigned a PRBS code up to a run length of $2^{\mathrm{L}-1}$ ( $\mathrm{L}$ is number of bits of LFSR used) and modified accordingly. Figure 2 shows the portion of modified PWM in red colour with PRBS implemented on it.

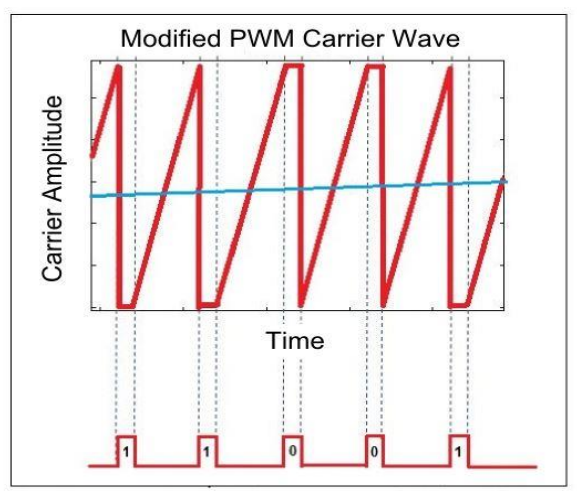

Figure 2: PRBS implementation on PWM: Modified Carrier wave with '1 10001 ' code

For a PRBS code ' 1 ', the carrier wave is set to the minimal value of the carrier signal, regardless of the amplitude of the reference signal and for code ' 0 ', the carrier wave is set to the maximal value of the carrier signal, regardless of the amplitude of the reference signal so that PWM never triggers in that time period. The consequence of this carrier modification is that for switching cycles correspondingto code ' 1 ', the pulse duration is widened and for code ' 0 ' the pulse duration is slightly narrowed. So, by giving small amount of energy required for identification, these variations from the original carrier are minimal and generate low amplitude, broad spectrum harmonics [8].

Grid impedance spectrum identification is done by measuring the output voltage and current with all harmonics and inter-harmonics and then obtaining ratio of their Fourier transform. The harmonics in the output voltage and current for normal PWM generation are mainly low frequency harmonics and switching harmonics. The majority of the switching harmonics are filtered at the output of the inverter and remaining low frequency harmonics are low amplitude voltage and current harmonics which can't provide broad range grid impedance spectrum. During the PRBS injection the spectrum of the PRBS is overlapped over the naturally generated harmonics which increases the possibility of broad spectrum grid impedance measurement. The grid impedance spectrum at PCC with all harmonics and inter- 
harmonics is thus obtained by the Fourier transform of the voltage and current at the PCC [8].

$$
Z(f)=\frac{D F T(V(t))}{D F T(I(t))}=R g r i d+j . X g r i d
$$

Where 'DFT' denotes the Discrete Fourier Transform of time domain measurement of voltage $\mathrm{V}(\mathrm{t})$ and current $\mathrm{I}(\mathrm{t})$ at PCC. Therefore, by aligning and synchronizing the PRBS codes with the fundamental frequency and performing a Fourier transform over the complete cycle render results containing minimum spectral leakage [8]. The next section describes the implemented test setup.

\section{Test setup implementation}

The test setup uses NI Single-Board RIO General Purpose Inverter Control (GPIC), which is programmed using LabVIEW and periodically generates PRBS. This section describes the hardware and software implementation done.

\section{A. Hardware}

The general overview of the system implemented is shown in Figure 3.

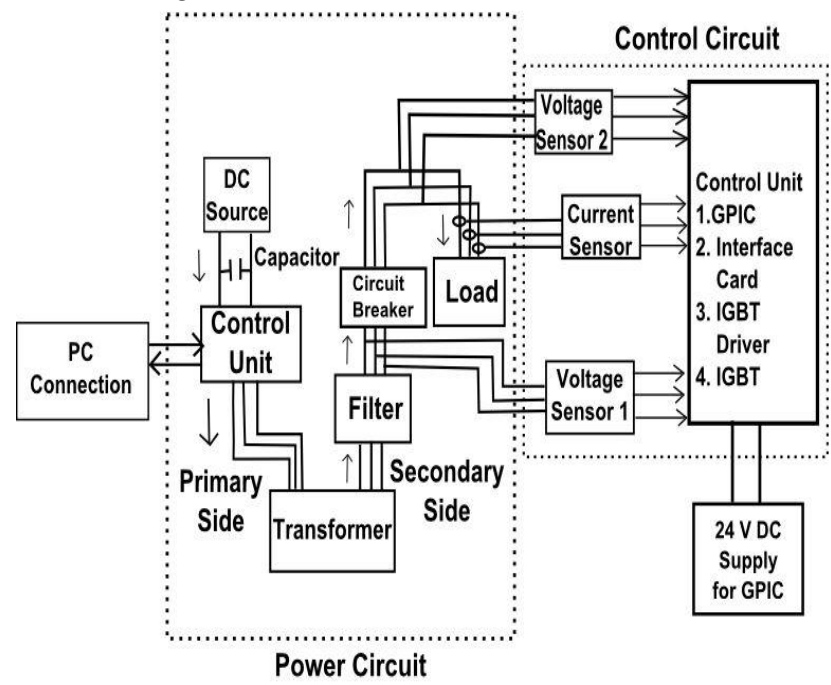

Figure 3: General overview of the System

The overall hardware design of Inverter has following subsystems:

DC Power Supply: The DC power supply is variable power source which has been used for simulating Distributed Energy Resource connected to the inverter. The DC power supply is a SM660-AR-11 with maximum output of $3300 \mathrm{~W}$ and voltage and current range 0-330 V and 0-11 A respectively.

Electrolyte Capacitor: It is used as a DC link between the DC power supply and inverter to prevent large transients generated at the output side of the inverter from reaching back to the DC supply and to act as a buffer for smoothing out DC voltage variations. Electrolyte Capacitor with capacitance of $400 \mu \mathrm{F}$ has been used as the DC link capacitor with maximum rated voltage and current, $1100 \mathrm{~V}$ (DC) and 40 A respectively.
Control Unit: The control unit consists of GPIC from National Instruments (NI), Interface Card, IGBT Driver and IGBT. The general overview of the control unit is shown in Figure 4.

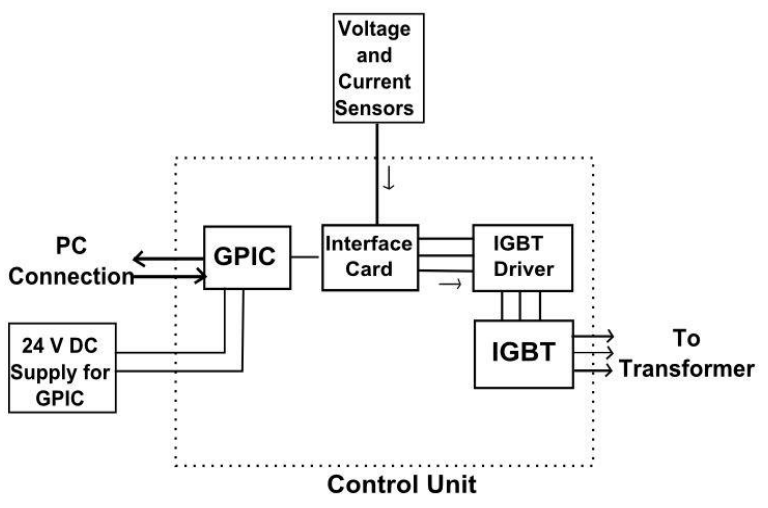

Figure 4: General overview of the control unit

NI Single-Board RIO GPIC is a typical stack of NI sbRIO-9606 (Single-Board RIO) control and monitoring system, NI GPIC RIO mezzanine card and custom interface board which is configured as per the requirement of the user. GPIC is programmed using a comprehensive NI LabVIEW graphical system design toolchain that includes simulation which make digital computing and compiling quite user friendly. The interface card takes the signal from the GPIC and appropriately delivers it to IGBT drivers for switching of IGBTs. Thus, it allows control of IGBT drivers which control IGBTs for generating $\mathrm{AC}$ output voltage. It is connected to a base board with IGBT driver which contains all necessary components for optimal and safe driving of IGBT modules. The gate signals produced are used for switching the IGBTs which are connected to variable power DC supply described earlier. The 3 phase AC output at the IGBTs is then connected to the primary side of the Transformer.

Transformer: The transformer used in this work is a 5 $\mathrm{kVA}$, Dzn0 type, air core step up transformer and is used to provide isolation of the output neutral from the source, to step up the output voltage at IGBTs and to provide impedance that limits fault current or acts as a noise filter. The transformer is designed to work for with a PWM switching frequency upto $16 \mathrm{kHz}$.

Output Filter: The LCL Filter used reduces the harmonics in the current generated by IGBTs switching and obtain low current distortions. It has a maximum rated operating voltage of $480 \mathrm{~V}$ with 56 A per phase for $50 \mathrm{~Hz}$ frequency.

Circuit breaker: A 4-pole circuit breaker from is used to connect or disconnect the inverter from the load. The maximum voltage and current rating are $6000 \mathrm{~V}$ and $15 \mathrm{~A}$.

Voltage and Current Sensors: Two voltage sensors and one current sensor are connected in the inverter circuit to measure and to control the output voltage and current of the inverter. Voltage sensors are connected before and after the breaker and measured voltages are sent into the 
GPIC for PWM generation. Current is measured after the breaker. Figure 5 shows the inverter cabinet with all hardware components implemented.

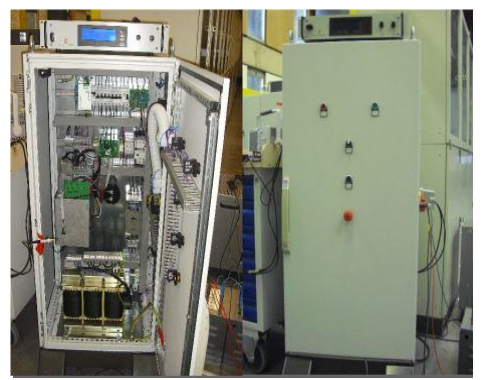

Figure 5: Lab Inverter Setup

\section{B. Software}

All the simulations of the system and the control were developed using LabVIEW, which is the system design software created by National Instruments. The main program is called VI (Virtual Instrument) and it can have a number of Sub-VIs. The GPIC used in this paper for inverter control has three different programming levels:

Host Computer (highest level): Provides user an interface for programming and monitoring of the system.

Real-Time (medium level): Executes the control algorithm received from Host Computer and sends the data to the FPGA level for processing. The processed data is then received at Real-Time level and communicated to the Host Computer.

FPGA level (low level): Does simple processing of the data, and sends out the control command. It is also the fastest, since processing is done in hardware rather than software.

When the program is running, each level does its own job. The actual FPGA and Real Time programming done has been briefly described.

\section{FPGA programming}

Inside the FPGA, the inverter control is implemented using 5 different control loops:

Analog input loop: Analog input loop reads simultaneously the instantaneous values at the output of the inverter from the interface card with analog input pins.

PWM generation Loop: Generates high speed PWM signals based on the outputs of the control loop. The sawtooth waveform and PRBS is generated with a Sub-VI and is started by a Restart PRBS function that injects on the sawtooth waveform for a limited cycles depending upon the Iteration Stop value set and initial seed value specified. The loop also has a Sub-VI which marks the starting of the PRBS injection. Thus, the PRBS generation takes place only when the voltage of Phase A is zero. An 11-bit LFSR is implemented with a PRBS sequence length of 2047 and has 4 taps so that it can be used for all LFSR upto 16 bit. The PRBS generator generates modified sawtooth with PRBS at 4 different magnitudes depending on the selection of the Binary Selector switches provided for the control and PRBS code generated i.e. ' 0 ' or ' 1 '. Where Sequence 1 is the PRBS with lowest magnitude and Sequence 4 is the PRBS with highest magnitude.Sawtooth waveform for PWM and modified PWM is generated using LabVIEW FPGA DDS (Direct Digital Synthesis) generator. The FPGA DDS generator generates repetitive waveforms with high degree of frequency and phase control. The reference waveform to be generated is specified in the look up table in FPGA DDS generator which is a straight line when PRBS is not activated. Figure 6 shows table preview of the look up table for sawtooth waveform generation.

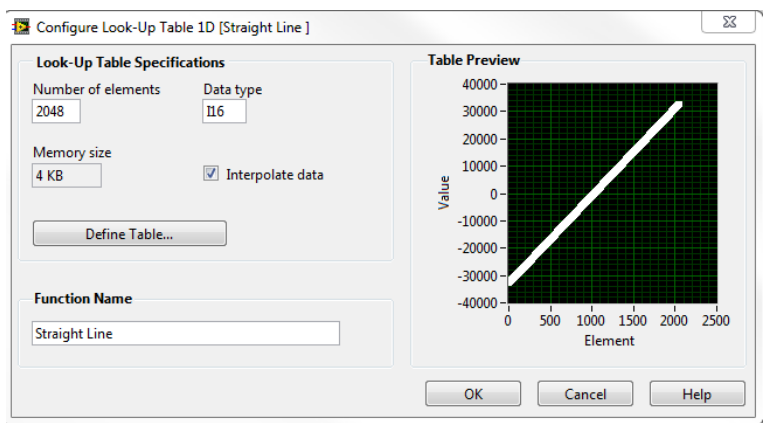

Figure 6: Configuring the sawtooth lookup table

Similarly the lookup table for the Sequence 1 to Sequence 4 has been implemented. When the PRBS is activated, the output waveform is generated depending on the code value generated by the PRBS generator and binary selector switch configuration selected. Figure 7 shows a table preview of the lookup table implemented for PRBS code ' 0 ' and PRBS code ' 1 ' with PRBS sequence 2 selection.
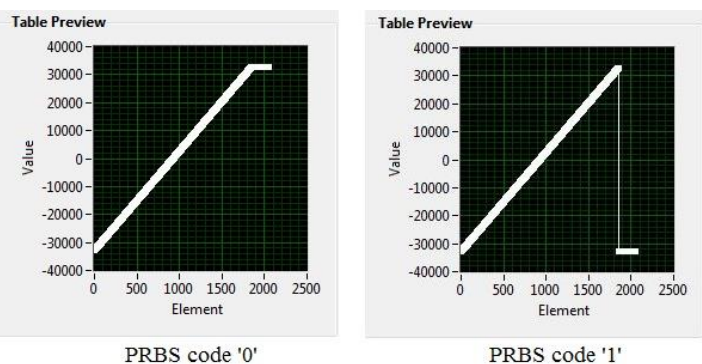

Figure 7: Table preview of the lookup table for PRBS code ' 0 ' and ' 1 '

The waveform generation operates at $12.8 \mathrm{kHz}$. The sawtooth waveform generated is then compared with three phase sine voltage signal generated by the control loop for PWM generation. The PWM generated controls the switching of IGBTs.

Reference Sine: The loop generates a reference three phase sine signal which is the signal that is desired at the output and used in analysis loop to synchronize the output voltage of the inverter with the desired signal.

Analysis Loop: The analysis loop is synchronized with Analog Input Loop and computes the coordinate transformations, RMS value of voltage and active and reactive power of the inverter.

Control Loop: Uses PID control algorithm in the $\mathrm{d}-\mathrm{q}$ reference frames to control voltage. 


\section{Real-Time Programming}

In the FPGA the memory is not endless so each of the memories, logic blocks etc. should be carefully used. In order to reduce the stress on FPGA and to increase its reliability the major complex operations are implemented in Real-Time. The Real-Time VI implemented has a front and back panel. The front panel is used for user interaction and is used for display and control and in the back panel the actual programming has been done.

\section{Results}

A three phase inductive load of $80 \mathrm{mH}$ is connected to the output of the inverter and with the grid impedance identification technique discussed the impedance spectrum for the inductor is identified.

For the impedance spectrum measurement inverter output is set to AC voltage of $10 \mathrm{~V}$ with DC power supply to 50 V. An 11-bit PRBS code on each phase is injected with the initial seed value '10100110000' and voltage and current values are measured only for Phase in our experiment. PRBS is injected for sequence length of 2047 which is approximately $160 \mathrm{~ms}$ with PRBS settings at width Sequence 2. The Voltage is measured across Phase $\mathrm{A}$ and neutral of the inductor and current is measured by measuring voltage across a shunt resistor of $1 \Omega$. The measurement data is collected through an oscilloscope and then Fourier transform of voltage and current is then done in MATLAB. Figure 8 shows the inverter with inductive load used for the experiment.

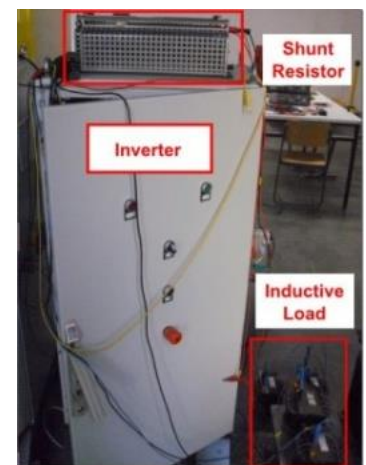

Figure 8: Inverter with Inductive Load and Shunt Resistor

A summary of the system parameters for the experimental is listed in the table below:

Table 1: System Parameters

\begin{tabular}{|l|l|}
\hline PRBS code polynomial & $\boldsymbol{x}^{\mathbf{1 1}}+\boldsymbol{x}^{\mathbf{9}}+\boldsymbol{x}^{\mathbf{6}}+\boldsymbol{x}^{\mathbf{5}}+\mathbf{1}$ \\
\hline Sampling Frequency & $100 \mathrm{kHz}$ \\
\hline Carrier Frequency & $12.8 \mathrm{kHz}$ \\
\hline Codes per fundamental cycle & 256 \\
\hline PRBS carrier duty cycle & $10 \%$ \\
\hline Load Parameters & $1 \Omega, 80 \mathrm{mH}$ \\
\hline DC Voltage Source & $50 \mathrm{~V}$
\end{tabular}

With these system settings the voltage and current waveform seen on the oscilloscope at the output of the inverter is shown in Figure 9. The yellow plot corresponds to the voltage and pink to the current. The scope plots shown are not filtered and data from these plots has been used for grid impedance spectrum identification. The harmonic content is partially due to the switching frequency, partially due to the PRBS code injected.

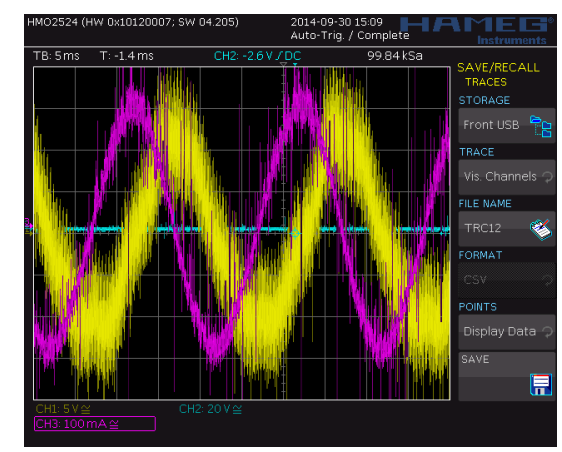

Figure 9: Inverter Output Voltage and Current

The results were obtained by PRBS injection using Sequence 2 for impedance spectrum identification of the inductive load. The voltage and current seen before and after the PRBS injected is shown in Figure 10. The yellow plot corresponds to the voltage, pink to the current and the blue line shows the period for which the PRBS is injected.

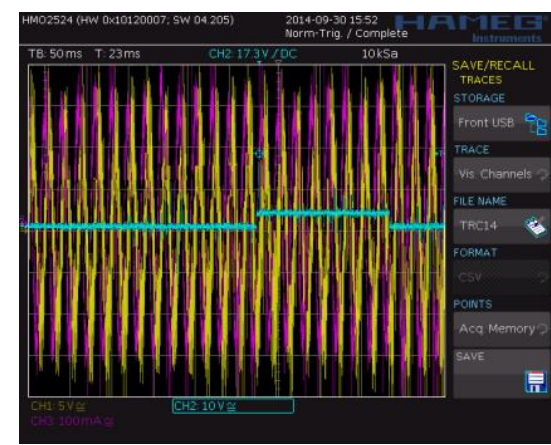

Figure 10: Voltage and Current Waveform with and without PRBS injection using Sequence 2

The data measured is then processed in MATLAB to plot the voltage and current spectrum when PRBS is injected and to plot the impedance spectrum upto a frequency of $1250 \mathrm{~Hz}$. The frequency of $1250 \mathrm{~Hz}$ has been chosen as cut-off because as per EN50160 [9] the harmonic orders up to 25 of fundamental frequency are considered as principally informative. The voltage and the current spectrum when PRBS is injected are shown in Figure 11 and Figure 12.

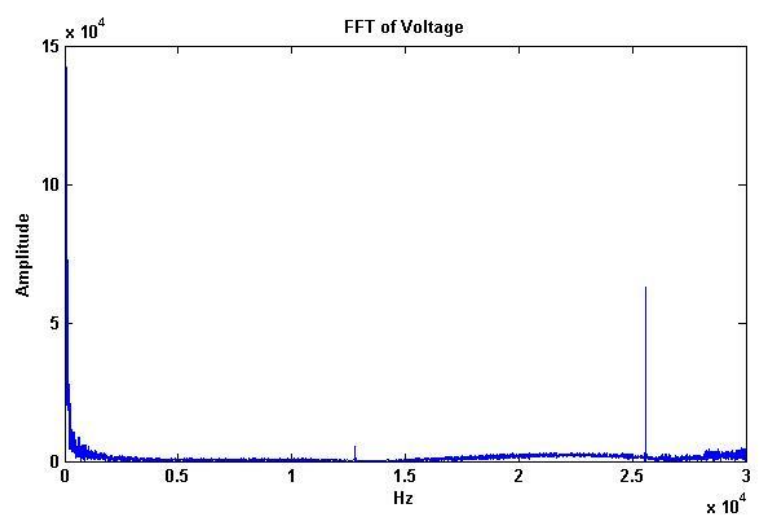

Figure 11: Voltage spectrum during PRBS injection 


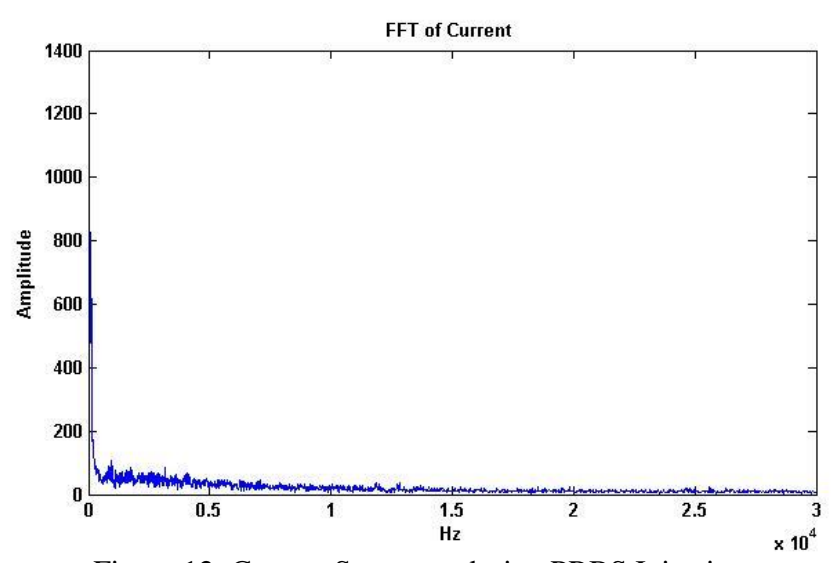

Figure 12: Current Spectrum during PRBS Injection

The impedance spectrum obtained from the voltage and current spectrum for the inductive load for PRBS sequence 2 is shown in Figure 13. The blue plot corresponds to the theoretical impedance spectrum and the red plot corresponds to the estimated impedance spectrum obtained by PRBS injection.

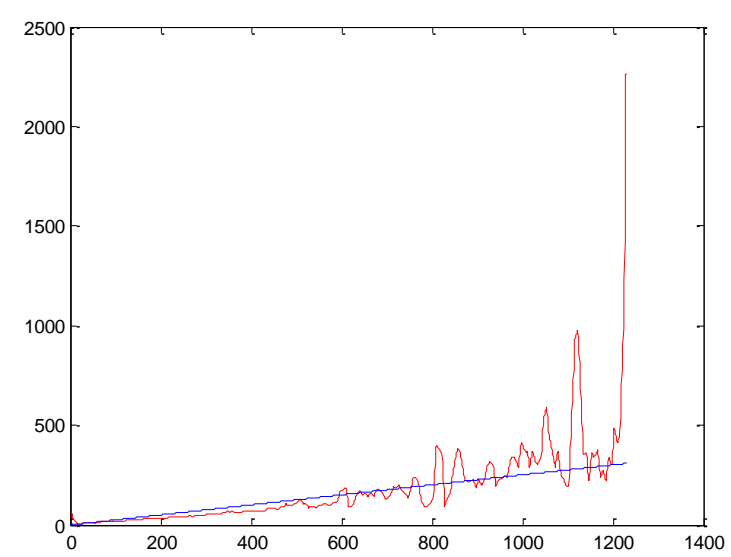

Figure 13: Impedance Spectrum Estimation for Inductive Load

Voltage spectrum and Current spectrum observed here exhibit the PRBS characteristics which mean that the proposed technique is implemented correctly on the inverter. Also the calculated impedance spectrum is close to the theoretical impedance spectrum of the inductive load. Also despite of the system noise while taking measurements through oscilloscope impedance measurement has not been affected which is attributed to correlation properties of the PRBS that makes it resilient to noise. In order to improve results, and alleviate the spectral instability of the PRBS, the data has been averaged over $20 \mathrm{~Hz}$ wide frequency bins. This has a smoothing effect on the obtained plot, and dampens the slight mismatches between Voltage and current spectrums. The results obtained are very satisfying up to $500 \mathrm{~Hz}$. On higher frequencies, the results are less accurate, mainly due to the low SNR of the PRBS injected harmonics. Nevertheless, the results are acceptable, but improvements can be obtained either by increasing the PRBS strength on higher frequencies, using a higher order LFSR, or by widening the frequency bins over higher frequencies.

\section{Conclusions}

The results obtained confirm that the proposed technique estimates the equivalent grid impedance over a significant frequency range with high resolution on a real time basis. It also provides a high degree of flexibility and is easy to implement with no additional hardware requirements. Several points for improvement are envisaged for further research. The modified PWM inverter hardware proposed in this research has output voltage limitations due to the DC power supply source used. A possible future work could be the implementation of a better DC power supply source to produce higher voltages at the output of the inverter. Though for the different power level, the control will be practically identical but with the increase of voltage there is possibility of decreasing the impedance estimation error. Finally, after verification of this novel method of online grid spectrum identification technique the future research aims on implementing prototype of an island grid in the SnT Netpower lab by connecting two inverters and then using PRBS for determining the impedance parameters between them in order to verify that the proposed technique is robust to a realistic environment and would represent a promising grid monitoring and diagnostic tool.

\section{Acknowledgement}

This work was conducted at the "Interdisciplinary Centre for Security, Reliability and Trust" (SnT) at the University of Luxembourg in collaboration with CREOS S.A., the Luxemburgish utility provider. It is supported by the National Research Fund, Luxembourg (SR/4881120).

\section{References}

[1] "Policy uncertainty threatens to slow renewable energy momentum," International Energy Agency, Paris, MediumTerm Renewable Energy Market Report 2014.

[2] L. Asiminoaei, E. Teodorescu, F. Blaabjerg, U. Borup, "Implementation and Test of an Online Embedded Grid Impedance Estimation Technique for PV Inverters, IEEE Transaction on Industrial Electronics, August 2005

[3] H. Langkowski, T Thanh, K. Dettmann, D. Schulz, „Grid Impèedance Determination - Relevancy for GRid Integration of Renewable Energy Systems“, IEEE 35th Annual Industrial Electronics IECON '09, November 2009

[4] K. De Brabandere, B. Bolsens, J. Van den Keybus, A. Woyte, J. Driesen, R. Belmans, "A Voltage and Frequency Droop Control Method for Parallel Inverters", IEEE 35th Annual Power Electronics Specialists Conference, 2004

[5] J. Sun, "Impedance-Based Stability Criterion for GridConnected Inverters", IEEE Transactions on Power Electronics, November 2011

[6] R.N. Mutagi, "Pseudo Noise Sequences for Engineers", IEEE Electronics and Comm. Eng. Journal, April 1996

[7] H. Okawara. (2013, June) ADVANTEST Corporation website. [Online]. www.advantest.com

[8] S. Neshvad, J. Sachau, and S. Chatzinotas, "Online Determination of Grid Impedance Spectrum through Pseudo-Random Excitation of a Pulse Width Modulator," Renewable Energy and Power Quality Journal, April 2014.

[9] EN 50160 , Power Quality Guide, "Merkmale der Spannung in öffentlichen Elektrizitätsversorgungsnetzen“ August 2007 